\title{
Clinical Outcomes for PD-1 Inhibitor Plus Chemotherapy as Second-Line or Later Therapy Compared to PD-1/PD-L1 Inhibitor Alone in Advanced Non-small-cell Lung Cancer
}

\author{
Xiaoyang Zhai ${ }^{1}$, Xuquan Jing ${ }^{1}$, Ji Li ${ }^{1}$, Yaru Tian ${ }^{1,2}$, Shuhui Xu' ${ }^{1}$, Min Wang ${ }^{1}$ and Hui Zhu ${ }^{1 \star}$ \\ ' Department of Radiation Oncology, Shandong Cancer Hospital and Institute, Shandong First Medical University \\ and Shandong Academy of Medical Sciences, Jinan, China, ${ }^{2}$ Department of Radiation Oncology, Shandong Cancer \\ Hospital and Institute Affiliated of Shandong University, Jinan, China
}

OPEN ACCESS

Edited by:

Kartik Sehgal,

Beth Israel Deaconess Medical Center and Harvard Medical School,

United States

Reviewed by:

Andreas Saltos,

Moffitt Cancer Center, United States Hirva Mamdani,

Wayne State University, United States

${ }^{*}$ Correspondence:

Hui Zhu

drzhuh@126.com

Specialty section:

This article was submitted to

Thoracic Oncology,

a section of the journal

Frontiers in Oncology

Received: 27 April 2020 Accepted: 04 September 2020 Published: 30 September 2020

Citation:

Zhai X, Jing X, Li J, Tian Y, XU S, Wang $M$ and $Z$ hu $H$ (2020) Clinical Outcomes for PD-1 Inhibitor Plus Chemotherapy as Second-Line or Later Therapy Compared to PD-1/PD-L1 Inhibitor Alone in Advanced Non-small-cell Lung Cancer. Front. Oncol. 10:556275. doi: 10.3389/fonc. 2020.556275
Background: Programmed death-1 (PD-1)/programmed death-ligand 1 (PD-L1) inhibitor monotherapy has been approved as second-line or later therapy in advanced non-small-cell lung cancer (NSCLC). The study aimed to compare the clinical outcomes of PD-1 inhibitor plus chemotherapy with PD-1/PD-L1 inhibitor monotherapy as second-line or later therapy in advanced NSCLC.

Methods: The clinical data of patients with advanced NSCLC who received PD-1/PDL1 inhibitors as second-line or later line therapy was retrospectively collected. Patients were assigned to one of the two groups according to the therapeutic modality used: PD1/PD-L1 inhibitor monotherapy group or PD-1 inhibitor plus chemotherapy combination therapy group. Disease control rate (DCR), progression-free survival (PFS), and overall survival (OS) were evaluated between the two groups. The prognostic effect of the derived neutrophil-to-lymphocyte ratio (dNLR) and lactate dehydrogenase (LDH) on the outcomes was also evaluated.

Results: From April 2017 to October 2019, a total of 84 patients were enrolled in the current study. Twenty-six patients (PD-1 inhibitor, $n=25$; PD-L1 inhibitor, $n=1$ ) received PD-1/PD-L1 inhibitor monotherapy, and fifty-eight patients received PD-1 inhibitor plus chemotherapy. The chemotherapy regimens used were as follows: liposome paclitaxel ( $n=15)$; nab-paclitaxel $(n=12)$; docetaxel $(n=9)$; pemetrexed $(n=6)$; and others $(n=16)$. The DCR and OS were not significantly different between the two groups. The PFS of the monotherapy group was longer than that of the combination therapy group (mPFS: 9.6 vs. 4.6 months, $P=0.01$ ). Univariate and multivariate analyses suggested that LDH and sex were independent prognostic factors of PFS. In the second-line therapy subgroup of 38 patients, OS and PFS were not significantly different between the two groups. In the subgroup of 46 patients treated beyond the 2nd line, the monotherapy group had a longer PFS (mPFS: 9.6 vs. 4.2 months, 
$P=0.01)$. The incidence of any-grade adverse events was not significantly different between the monotherapy group and the combination therapy group (19.2 vs. $18.9 \%$, $P=1.000$ ). One patient in the PD-1 inhibitor plus chemotherapy group died of immune-related pneumonitis.

Conclusion: The clinical outcomes of PD-1 inhibitor plus chemotherapy as second-line or later therapy were similar to those of PD-1/PD-L1 inhibitor alone in advanced NSCLC.

Keywords: non-small-cell lung cancer, PD-1, PD-L1, second-line or later therapy, immune-related adverse events

\section{INTRODUCTION}

Lung cancer is still the malignant tumor with the highest incidence and mortality worldwide (1). Non-small-cell lung cancer (NSCLC) accounts for approximately 80 to $85 \%$ of all lung cancers (2). Despite recent advances in treatment, advanced NSCLC still has a poor prognosis. Especially for patients whose disease progresses after first-line therapy, alternative drugs are limited. Docetaxel is used as the second- and third-line therapy, with a median overall survival of only 7.5 months and survival rates of $32 \%$ at 1 year $(3,4)$. The antibodies that target programmed death-1 (PD-1) and programmed death-ligand 1 (PD-L1) have been approved for second-line or subsequent therapy, showing overall survival (OS) benefits over docetaxel.

The approval of these PD-1/PD-L1 antibodies was mainly based on the results of the CheckMate 017, CheckMate 057, KEYNOTE 010 and OAK trials (5-8). In these trials, nivolumab, used as second-line therapy, showed a 5 -year survival rate above fourfold higher than docetaxel ( 13 vs. $3 \%$, hazard ratio $0.68,95 \%$ CI 0.59-0.78) (9). Compared with docetaxel, pembrolizumab showed a median OS of 10.4 vs. 8.5 months (HR 0.71, 95\% CI $0.58-0.88$ ) at the approval dose of $2 \mathrm{mg} / \mathrm{kg}$ in NSCLC patients whose PD-L1 expression is $1 \%$ or above in tumor cells (7). Moreover, atezolizumab also showed longer OS (13.8 vs. 9.6 months, HR $0.73,95 \%$ CI $0.62-0.87$ ) than docetaxel in patients with previously treated advanced NSCLC (8).

Chemotherapy, as a conventional therapy mode, has a synergistic antitumor effect with the PD-1/PD-L1 inhibitor. Chemotherapy can enhance the immune response regained by PD-1/PD-L1 inhibitors by a series of underlying mechanisms, such as strengthening the sensitivity of tumor cells to the lysis effect of cytotoxic T lymphocytes (CTLs) (10), increasing the immunogenicity of tumor cells (11), reducing immunosuppressive cells by inducing cell apoptosis $(12,13)$, and promoting the antitumor immune response by changing the tumor microenvironment (14). PD-1/PD-L1 inhibitors combined with chemotherapy, approved as a first-line therapy, has given more benefit to extensive population $(15,16)$.

Abbreviations: AEs, adverse events; ALK, anaplastic lymphoma kinase; CTCAE, Common Terminology Criteria for Adverse Events; CTL, cytotoxic T lymphocyte; DCR, disease control rate; dNLR, derived neutrophil to lymphocyte ratio; EGFR, epidermal growth factor receptor; HPD, hyperprogressive disease; HR, hazard ratio; KPS, Karnofsky performance status; LDH, lactate dehydrogenase; NSCLC, non-small-cell lung cancer; OS, overall survival; PD-1, programmed death-1; PDL1, programmed death-ligand 1; PFS, progression-free survival; RECIST, Response Evaluation Criteria in Solid Tumors; SD, stable disease; TKI, tyrosine kinase inhibitor; TPS, tumor proportion score; ULN, upper limit of normal.
However, the efficacy and safety of PD-1/PD-L1 inhibitors plus chemotherapy as second-line or later therapy remained unclear. Therefore, we performed this retrospective study to explore the clinical efficacy and safety of second-line or later therapy with PD-1/PD-L1 inhibitors plus chemotherapy in advanced NSCLC patients and to compare the clinical outcomes of PD-1/PD-L1 inhibitors plus chemotherapy with PD-1/PD-L1 inhibitor monotherapy as second-line or later therapy further.

\section{MATERIALS AND METHODS}

\section{Patients}

We retrospectively reviewed the records of advanced NSCLC patients who received a PD-1/PD-L1 inhibitor as second-line or later therapy in Shandong Cancer Hospital and Institute (Jinan, Shandong, China) between April 2017 and October 2019. Eligible patients were histologically or cytologically diagnosed with NSCLC, had a Karnofsky Performance Status (KPS) score $\geq 80$, and had measurable lesions. Patients harboring epidermal growth factor receptor (EGFR) mutations or anaplastic lymphoma kinase (ALK) fusions and those who suffered from failure of tyrosine kinase inhibitor (TKI) therapy were also enrolled in the study. Patients with autoimmune disease, interstitial lung disease and immunosuppression and those who were previously treated with a cytotoxic T-lymphocyte antigen-4 (CTLA-4) inhibitor, PD-1/PD-L1 inhibitor or immunosuppressant were excluded. Patients without response evaluation because of fewer than 2 cycles or loss to follow-up were also excluded. The study was approved by the Ethics Committee of Shandong Cancer Hospital and Institute. All procedures involving patients conformed to the principles outlined in the Declaration of Helsinki.

\section{Treatments}

The patients were divided into the PD-1/PD-L1 inhibitor monotherapy group and the PD-1 inhibitor plus chemotherapy combination therapy group based on their treatment modality. The PD-1/PD-L1 inhibitors in the monotherapy group included sintilimab, pembrolizumab, nivolumab, camrelizumab, atezolizumab and RB004. The PD-1 inhibitors in the PD1 inhibitor plus chemotherapy group included sintilimab, pembrolizumab, nivolumab, camrelizumab, and toripalimab. The chemotherapy regimens involved liposome paclitaxel, nab-paclitaxel, docetaxel, pemetrexed, and others. The PD-1/PD-L1 inhibitors and chemotherapy drugs were all administered intravenously. The therapeutic schedule for each 
patient was decided by the attending physicians based on the efficacy of the previous therapy and the patient's physical condition and intentions.

\section{Assessment of Response and Toxicity}

The response evaluation of tumors was based on the Response Evaluation Criteria in Solid Tumors (RECIST) version 1.1. Evaluation was performed routinely every 6-8 weeks after starting treatment with the PD-1/PD-L1 inhibitor. Adverse events (AEs) were assessed according to the National Cancer Institute Common Terminology Criteria for Adverse Events (CTCAE) version 4.0. AEs that occurred during hospitalization were registered and graded by their attending doctor timely, while AEs that occurred outside the hospital were mainly based on patients' initiative report.

\section{Endpoints}

The primary endpoint was OS, which was defined as the time interval from the treatment initiation of the PD-1/PD-L1 inhibitor to death caused by any reason or the last known followup. The secondary endpoints were progression-free survival
(PFS), disease control rate (DCR), and AEs. PFS was measured as the time interval from the initiation of PD-1/PD-L1 inhibitor treatment to tumor progression, death from any cause or the last known follow-up. According to RECIST version 1.1, objective response rate (ORR) refers to the proportion of patients who had a complete or partial response, and the DCR refers to that of patients who had a complete or partial response or stable disease (SD). Moreover, we also collected the pretreatment (within 30 days before the first PD-1/PD-L1 inhibitor treatment)derived neutrophil to lymphocyte ratio (dNLR) and lactate dehydrogenase (LDH) to explore their prognostic effects on the outcome of advanced NSCLC patients. The dNLR is calculated by neutrophils/leukocytes minus neutrophils. $\mathrm{dNLR}>3$ and $\mathrm{LDH}>$ upper limit of normal (ULN) were defined as high $(17,18)$.

\section{Statistical Analysis}

All statistical analyses were performed using GraphPad Prism software version 8.0 (GraphPad Software, Inc., United States) and SPSS statistical software version 20.0 (IBM Corp., United States). The comparisons of patients' baseline characteristics, tumor

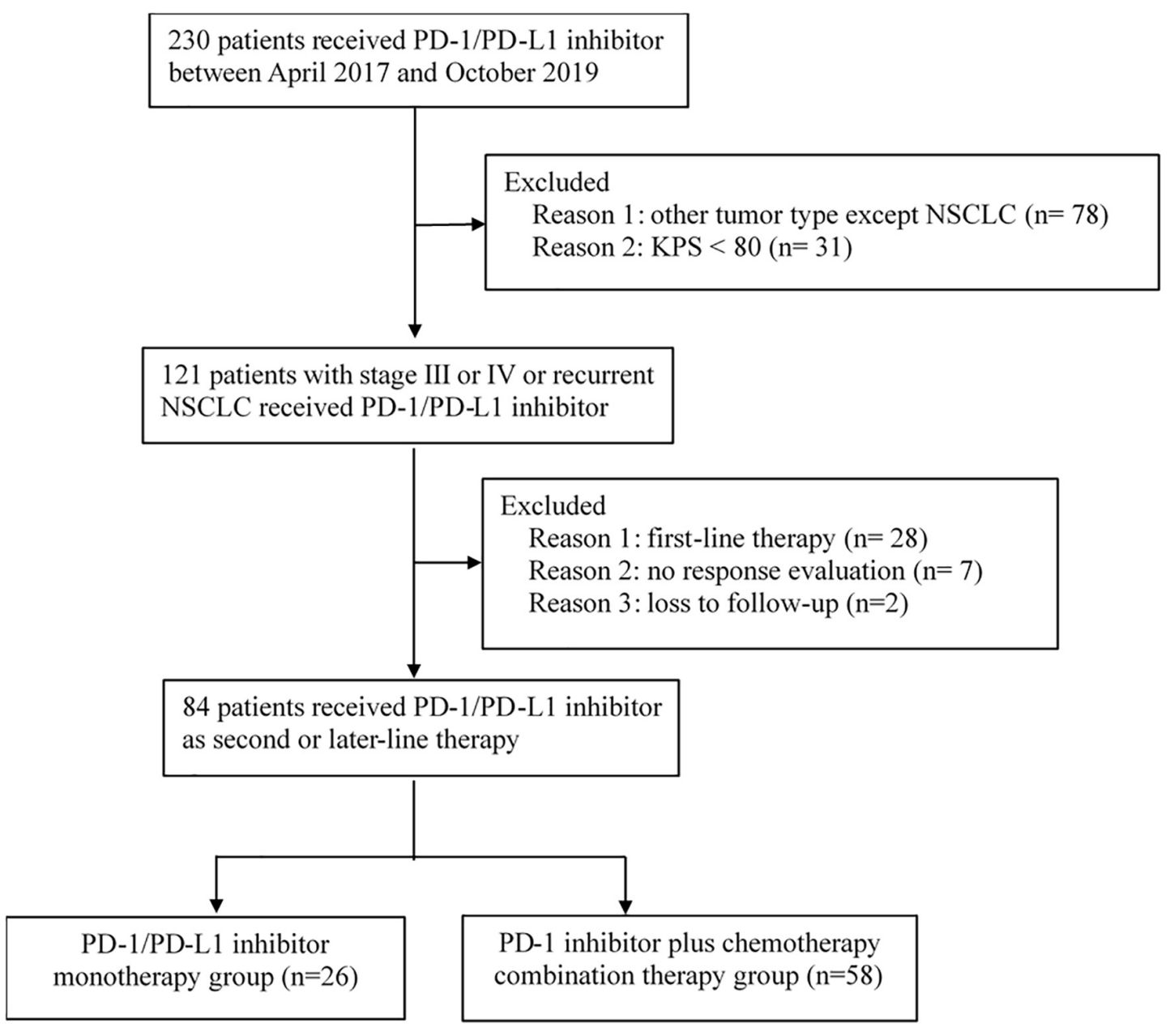

FIGURE 1 | Study diagram. 
response and AEs in the two groups were analyzed using the Chi-square test and Fisher's exact test. Univariate survival analysis was performed using the Kaplan-Meier method. Multivariate survival analysis was performed by a Cox proportional hazards model to evaluate the independent prognostic factors associated with improved survival. The Kaplan-Meier method was used to calculate OS and PFS. The difference in survival curves between the two groups was estimated by the log-rank test. Two-sided $P$ values $<0.05$ were considered statistically significant.

\section{RESULTS}

\section{Patient Characteristics}

Between April 2017 and October 2019, 230 patients received immunotherapy in our cancer center. 78 patients were excluded for other tumor types, and 31 patients were excluded for KPS $<80$. The total number of patients with stage IIIB or IV or recurrent NSCLC who received PD-1/PD-L1 inhibitor treatment was 121 . Twenty-eight patients receiving PD-1/PD-L1 inhibitor as first-line therapy and seven patients without response evaluation were excluded. The follow-up rate was $97.6 \%$, and two patients were lost to follow-up (Figure 1). As a result, a total of 84 patients who received PD-1/PD-L1 inhibitor as the secondline or later therapy were enrolled in the study. According to the therapeutic modality, there were 26 patients in the monotherapy group and 58 patients in the combination group. A total of $62.1 \%$ of patients in the combination group received taxane drugs, including liposome paclitaxel, nab-paclitaxel, and docetaxel. The last follow-up date was June 30, 2020. Fifty-three patients were still alive and thirty-one patients were died by the end of the follow-up. The median follow-up time was 11.4 months (range, 2.1-24.7 months) for all patients and 12.6 months (range, 8.824.7 months) for living patients. The baseline characteristics of all patients in the two groups are presented in Table 1. There were no differences in the distribution of most variables other than histologic features between the two groups. The rate of adenocarcinoma patients was $74.1 \%$ in the combination group and $42.3 \%$ in the monotherapy therapy group. The median age of the patients in the two groups was 62 years, and the age range was 29 to 81 years. Thirty-eight (45.2\%) patients had received second-line therapy, and 46 (54.8\%) patients had received third-line or later therapy. Twenty (23.8\%) patients had developed brain metastases, and 13 (15.4\%) patients had developed liver metastases. Forty-five (53.5\%) patients were never smokers, which is higher than that reported in clinical trials. Moreover, in our study, there were 9 patients $(10.7 \%)$ with tumors harboring EGFR mutations and 2 patients with tumors harboring ALK fusions.

Among the 84 patients, 24 patients had information available regarding the PD-L1 tumor proportion score (TPS). 33.3\% (8/24) patients had PD-L1 TPS scores that were less than 1\%, 25\% (6/24) had TPS scores between 1 and 50\%, and 41.6\% (10/24) had TPS scores greater than or equal to $50 \%$. Positive rates of tumor PD-L1 expression were balanced between the monotherapy group and the combination therapy group (Table 1).
TABLE 1 | Clinical features of patients with advanced NSCLC receiving second-line or later therapy in the monotherapy group or the combination therapy group.

\begin{tabular}{|c|c|c|c|c|c|}
\hline \multirow[t]{2}{*}{ Characteristic } & \multirow[t]{2}{*}{ Total } & \multicolumn{2}{|c|}{$\begin{array}{c}\text { Monotherapy } \\
\text { group }\end{array}$} & \multicolumn{2}{|c|}{$\begin{array}{l}\text { Combination } \\
\text { therapy group }\end{array}$} \\
\hline & & No. & $\%$ & No. & $\%$ \\
\hline
\end{tabular}

\section{Gender}

Male

Female

$\begin{array}{lllll}60 & 22 & 84.6 & 38 & 65.5\end{array}$

Age (years)

Range

Median

$<65$

$\geq 65$

KPS score

$\geq 90$

80-90

24

4

15.4

20

34.5

0.073

\section{Smoking status}

Current or former smoker

Never smoker

29-81 43-81

$62 \quad 60$

$57 \quad 17$

$27 \quad 9$

65.4

29-79

Histologic features

Adenocarcinoma

Squamous

Other

Line of therapy

Second

Third or later

Brain metastases

Yes

No

Liver metastases

Yes

No

Previous radiotherapy

Yes

Thoracic radiotherapy

Other or unknown

No

EGFR mutation

Yes

No

Unknown

$38 \quad 12$

34.6

62

40

69.0

18

31.0

0.745

$46 \quad 14$

$46.2 \quad 26$

44.8

55.2

0.910

$\begin{array}{lllll}39 & 13 & 50.0 & 26 & 44.8\end{array}$

$\begin{array}{lllll}45 & 13 & 50.0 & 32 & 55.2\end{array}$

0.660

$\begin{array}{lllll}54 & 11 & 42.3 & 43 & 74.1\end{array}$

$\begin{array}{lllll}25 & 13 & 50.0 & 12 & 20.7\end{array}$

$\begin{array}{lllll}5 & 2 & 7.7 & 3 & 5.2\end{array}$

0.011

$\begin{array}{lllll}38 & 14 & 53.8 & 24 & 41.4\end{array}$

$\begin{array}{lllll}46 & 12 & 46.2 & 34 & 58.6\end{array}$

0.289

PD-L1 expression

Positive

Negative

Unknown

LDH level ${ }^{*}$

$>$ ULN

$\leq$ ULN

dNLR level ${ }^{※}$

$>3$

$\leq 3$

Immunotherapy drugs

Sintilimab

Pembrolizumab $\begin{array}{lllll}20 & 4 & 15.4 & 16 & 27.6\end{array}$

$\begin{array}{lllll}64 & 22 & 86.6 & 42 & 72.4\end{array}$

0.225

20.7

$\begin{array}{lllll}13 & 1 & 3.8 & 12 & 20.7\end{array}$

$71 \quad 25$

96.2

46

79.3

0.056

$49 \quad 13$

50.0

36

62.1

20

16

193

$35 \quad 13$

50.0

37.9

0.300

12.1

65.5

22.4

0.172

50.0

13

15.5

10.3

74.1

0.481

46.6

$53.4 \quad 0.146$

29.3

70.7

0.693

18

38.5

35

60.3

17.2 
TABLE 1 | Continued

\begin{tabular}{|c|c|c|c|c|c|c|}
\hline \multirow[t]{2}{*}{ Characteristic } & \multirow[t]{2}{*}{ Total } & \multicolumn{2}{|c|}{$\begin{array}{l}\text { Monotherapy } \\
\text { group }\end{array}$} & \multicolumn{2}{|c|}{$\begin{array}{l}\text { Combination } \\
\text { therapy group }\end{array}$} & \multirow[t]{2}{*}{$P$} \\
\hline & & No. & $\%$ & No. & $\%$ & \\
\hline Nivolumab & 10 & 5 & 19.2 & 5 & 8.6 & \\
\hline Other checkpoint inhibitors & 14 & 6 & 22.1 & 8 & 13.8 & 0.204 \\
\hline
\end{tabular}

*Two patients with unknown dNLR and LDH were unevaluable. NSCLC, nonsmall-cell lung cancer; KPS, Karnofsky performance status; AJCC, American Joint Committee on Cancer; EGFR, epidermal growth factor receptor; $L D H$, lactate dehydrogenase; ULN, upper limit of normal; $d N L R$, derived neutrophil to lymphocyte ratio; $\chi 2$, Chi-square statistic.

TABLE 2 | Tumor response in patients with advanced NSCLC receiving second-line or later therapy in the monotherapy group or the combination therapy group.

\begin{tabular}{|c|c|c|}
\hline & $\begin{array}{l}\text { Monotherapy } \\
\text { group }(n=26)\end{array}$ & $\begin{array}{c}\text { Combination therapy } \\
\text { group }(n=58)\end{array}$ \\
\hline Objective response rate, $\mathrm{n}(\%)$ & 5 (19.2\%) & $9(15.5 \%)$ \\
\hline$P$ value & 0.832 & \\
\hline Disease control rate, $\mathbf{n}(\%)$ & $21(80.8 \%)$ & $43(74.1 \%)$ \\
\hline$P$ value & 0.509 & \\
\hline \multicolumn{3}{|l|}{ Best overall response, $\mathrm{n}(\%)$} \\
\hline Complete response & 0 & 0 \\
\hline Partial response & $5(19.2 \%)$ & $9(15.5 \%)$ \\
\hline Stable disease & $16(61.5 \%)$ & $34(58.6 \%)$ \\
\hline Progressive disease & $5(19.2 \%)$ & 15 (25.9\%) \\
\hline
\end{tabular}

$P R$, partial response; $S D$, stable disease; $P D$, progressive disease; $D C R$, disease control rate.

\section{Response}

Among all 84 patients, the ORR was $16.7 \%$, and the DCR was $76.2 \%$. The ORR was $19.2 \%$ in the monotherapy group and $15.5 \%$ in the combination therapy group $(P=0.832)$. The DCR was $80.8 \%$ in the monotherapy group and $74.1 \%$ in the combination therapy group $(P=0.509)$. The difference in the response rate between these two groups was not significant (Table 2).

\section{Survival}

The median OS in the monotherapy group and in the combination therapy group were not reached (NR). The OS rates at 1 year in the monotherapy group and combination therapy group were 76.5 and $58.6 \%$, respectively. OS was not significantly different between the PD-1/PD-L1 monotherapy group and the $\mathrm{PD}-1$ plus chemotherapy group among all 84 patients $(P=0.061$, Figure 2A). The median PFS was 9.6 months for the patients receiving monotherapy and 4.6 months for those receiving combination therapy. PFS was longer in the PD-1/PD-L1 inhibitor monotherapy group than in the PD-1 inhibitor plus chemotherapy group $(P=0.018$, Figure 2B). The PFS rates at 1 year in the monotherapy group and combination therapy group were 45.1 and $17.8 \%$, respectively.

In the subgroup of 38 patients receiving second-line therapy, the OS was not significantly different between the PD-1/PDL1 monotherapy group and the PD-1 plus chemotherapy group (median OS: NR vs. NR, $P=0.066$, Figure 3A). The OS rates at 1 year in the monotherapy group and combination therapy group were 85.7 and $59.4 \%$, respectively. The difference in PFS between the two groups was also not significant (median PFS: 8.7 vs. 6.9 months, $P=0.354$, Figure $3 B$ ). In the subgroup of 46 patients receiving treatment beyond second-line therapy, patients in the monotherapy group had a longer PFS than those in the combination therapy group (median PFS: 9.6 vs. 4.2 months, $P=0.019$, Figure 4B). The OS between the two groups was not significantly different (median OS: NR vs. 12.5 months, $P=0.441$, Figure 4A).

Additionally, the analysis showed that OS was significantly longer in patients with $\mathrm{LDH} \leq \mathrm{ULN}$ than in patients with $\mathrm{LDH}>\mathrm{ULN}$. The median OS was 11.6 months in the group of $\mathrm{LDH}>\mathrm{ULN}$ and $\mathrm{NR}$ in group of $\mathrm{LDH} \leq \mathrm{ULN}$ $(P=0.022$, Figure 5A). The median PFS was 4.2 months in patients of $\mathrm{LDH}>\mathrm{ULN}$ and 7.3 months in patients of LDH $\leq$ ULN. This difference was also significant $(P=0.016$, Figure 5B).

\section{Prognostic Factors}

The clinical characteristics of the patients were evaluated to determine their prognostic value for OS (Table 3). Univariate analysis indicated that age, sex, KPS score, smoking status, histologic features, line of therapy, brain metastases, liver metastases, previous radiotherapy, EGFR mutation status, dNLR, immunotherapy drugs and treatment mode were not associated
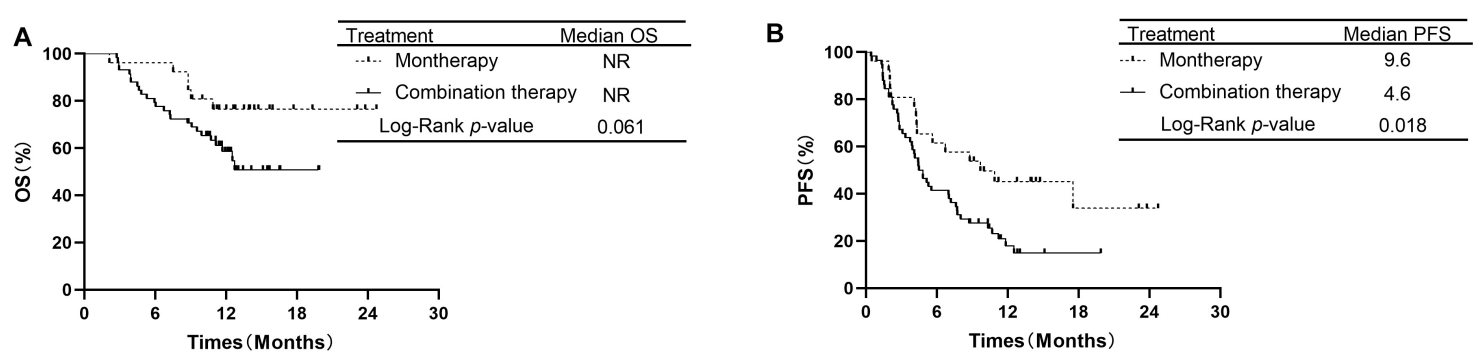

FIGURE 2 | Comparison of OS and PFS of patients with advanced NSCLC receiving second-line or later therapy between PD-1/PD-L1 inhibitor alone and PD-1 inhibitor plus chemotherapy. (A) Comparison of OS. (B) Comparison of PFS. NSCLC, non-small-cell lung cancer; PD-1, programmed death-1; PD-L1, programmed death-ligand 1; OS, overall survival; PFS, progression-free survival. 

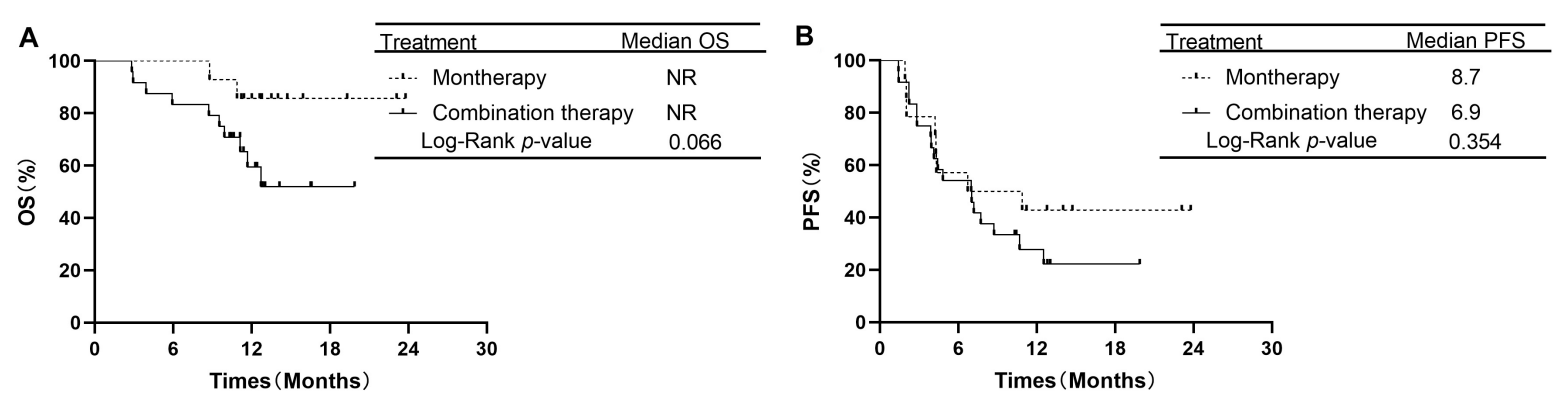

FIGURE 3 | Overall survival and progression free survival in subgroup analysis of second-line therapy between PD-1/PD-L1 inhibitor alone and PD-1 inhibitor plus chemotherapy. (A) Comparison of OS. (B) Comparison of PFS. NSCLC, non-small-cell lung cancer; PD-1, programmed death-1; PD-L1, programmed death-ligand 1; OS, overall survival; PFS, progression-free survival.
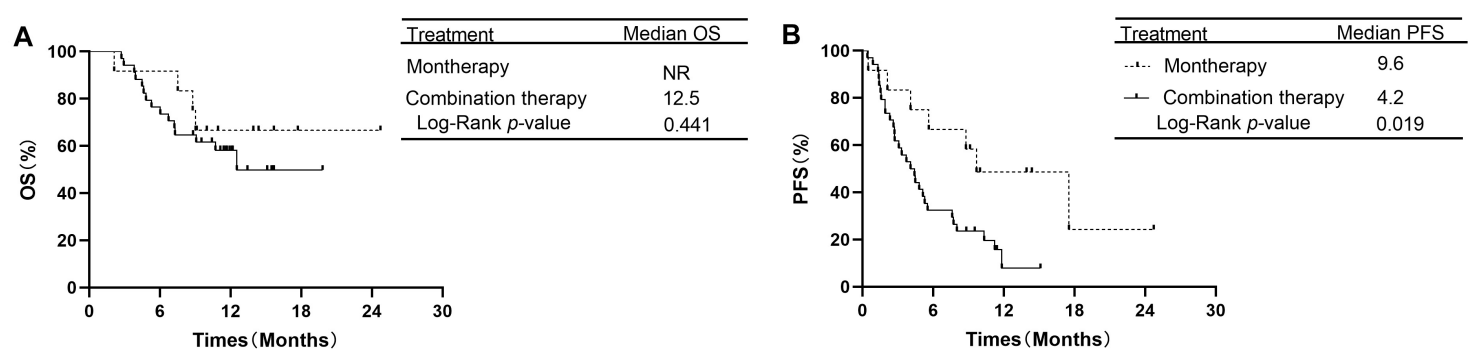

FIGURE 4 | OS and PFS in subgroup analysis of treatment beyond second-line therapy between PD-1/PD-L1 inhibitor alone and PD-1 inhibitor plus chemotherapy. (A) Comparison of OS. (B) Comparison of PFS. NSCLC, non-small-cell lung cancer; PD-1, programmed death-1; PD-L1, programmed death-ligand 1; OS, overall survival; PFS, progression-free survival.
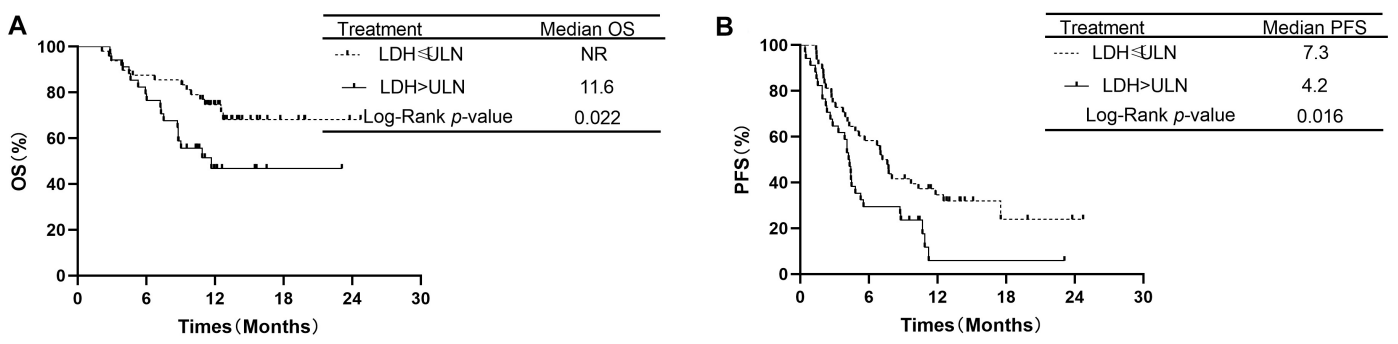

FIGURE 5 | Comparison of overall survival and progression-free survival of patients with LDH $\leq$ ULN vs. LDH $>$ ULN.

with survival. $\mathrm{LDH} \leq \mathrm{ULN}$ was associated with better OS than $\mathrm{LDH}>\mathrm{ULN}(P=0.022)$. However, multivariate analyses indicated that no clinical factors were associated with OS. For PFS, univariate analysis revealed that male sex, nonadenocarcinoma status, non-liver metastases, LDH $\leq$ ULN and monotherapy were significant favorable prognostic factors (Table 4). Multivariate analysis revealed that male sex $(P=0.035)$ and $\mathrm{LDH} \leq \mathrm{ULN}(P=0.043)$ were favorable prognostic factors for PFS.

\section{Toxicities}

The incidence of AEs is shown in Table 5. The rate of any grade AEs was $19.2 \%(5 / 26)$ in the PD-1 inhibitor monotherapy group and $18.9 \%(11 / 58)$ in the PD-1 plus chemotherapy group. The rate of grade 3-4 AEs was relatively higher in the combination group than in the monotherapy group (10.2 vs. $7.6 \%, P=1.000)$. However, the difference was not significant. The most common grade 3-4 AEs were pneumonitis in the monotherapy group and pneumonitis and myocarditis in the combination group. One patient $(3.8 \%)$ in the monotherapy group withdrew treatment because of pneumonitis, and 5 patients $(8.5 \%)$ in the combination group discontinued treatment because of pneumonitis, myocarditis, and diarrhea. One patient in the combination group died of immune-related pneumonitis.

\section{DISCUSSION}

Data from KEYNOTE 189, KEYNOTE 407, and the IMPOWER 150 trial indicated that chemotherapy combined with 
TABLE 3 | Univariate analysis and multivariate analysis of the prognostic factors for OS in patients with advanced NSCLC receiving second-line or later-line therapy.

\begin{tabular}{llllllllll}
\hline & \multicolumn{3}{c}{ Univariate analysis } & & \multicolumn{3}{c}{ Multivariate analysis } \\
\cline { 2 - 3 } \cline { 5 - 7 } Factors & MST (m) & $\times$ & 2 & $P$ & & HR & $95 \%$ Cl & $P$ \\
\hline
\end{tabular}

\section{Gender}

Male

Female

Age (years)

$<65$

$\geq 65$

KPS score

$\geq 90$

80-90

Smoking status

Current or former smoker

Never smoker

Histologic features

Adenocarcinoma

Non-adenocarcinoma

Line of therapy

Second

Third or later

Brain metastases

Yes

No

Liver metastases

Yes

No

Previous radiotherapy

Yes

No

EGFR mutation $*$

Yes

No

LDH

$>$ ULN

$\leq$ ULN

dNLR

$>3$

$\leq 3$

Immunotherapy drugs

Sintilimab

Pembrolizumab

Nivolumab

Other checkpoint inhibitors

Treatment mode

Monotherapy

Combination

*Indicates that the patients' EGFR mutation status was assessable. NSCLC, nonsmall-cell lung cancer; MST, median survival time; KPS, Karnofsky performance status; AJCC, American Joint Committee on Cancer; EGFR, epidermal growth factor receptor; $L D H$, lactate dehydrogenase; ULN, upper limit of normal; dNLR, derived neutrophil to lymphocyte ratio; $x 2$, Chi-square statistic.

immunotherapy $(15,19,20)$ had superior efficacy over conventional chemotherapy as a first-line therapy for advanced NSCLC, with median survival times of 22, 15.9, and 19.2 months
TABLE 4 | Univariate analysis and multivariate analysis of the prognostic factors for PFS in patients with advanced NSCLC receiving second-line or later-line therapy.

\begin{tabular}{|c|c|c|c|c|c|c|}
\hline \multirow[b]{2}{*}{ Factors } & \multicolumn{3}{|c|}{ Univariate analysis } & \multicolumn{3}{|c|}{ Multivariate analysis } \\
\hline & Median, m & $\times 2$ & $\boldsymbol{P}$ & HR & $95 \% \mathrm{Cl}$ & $P$ \\
\hline
\end{tabular}

Gender

Male

Female

7.1

Age (years)

$<65$

$\geq 65$

3.0

$\begin{array}{lllll}6.784 & 0.009 & 0.409 & 0.179-0.937 & 0.035\end{array}$

5.2

$\begin{array}{llllll}7.0 & 1.148 & 0.284 & 1.522 & 0.809-2.861 & 0.192\end{array}$

KPS score

$\geq 90$

80-90

\section{Smoking status}

Current or former smoker

Never smoker

$\begin{array}{lllll}0.720 & 0.396 & 0.683 & 0.314-1.489 & 0.338\end{array}$

Histologic features

Adenocarcinoma

Non-adenocarcinoma

\section{Line of therapy}

Second

Third or later

Brain metastases

Yes

No

5.5

4.8

$\begin{array}{lllll}0.774 & 0.379 & 0.811 & 0.471-1.396 & 0.449\end{array}$

6.9

4.4

4.4

$\begin{array}{llllll}10.3 & 8.800 & 0.003 & 1.574 & 0.814-3.043 & 0.177\end{array}$

6.9

$\begin{array}{llllll}4.8 & 1.594 & 0.207 & 0.668 & 0.386-1.156 & 0.150\end{array}$

4.0

$\begin{array}{llllll}6.9 & 0.791 & 0.374 & 0.843 & 0.413-1.719 & 0.638\end{array}$

Liver metastases

Yes

No

Previous radiotherapy

Yes

No

EGFR mutation*

Yes

No

LDH

$>$ ULN

$\leq$ ULN

dNLR

$>3$

$\leq 3$

Immunotherapy drugs

Sintilimab

Pembrolizumab

Nivolumab

Other checkpoint inhibitors

Treatment mode

Monotherapy

Combination therapy

3.7

$\begin{array}{llllll}6.7 & 6.016 & 0.014 & 0.716 & 0.354-1.448 & 0.353\end{array}$

4.4

$\begin{array}{llllll}6.7 & 0.000 & 0.993 & 0.792 & 0.444-1.410 & 0.428\end{array}$

4.0

5.2

1.8070 .179

4.2

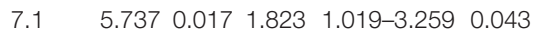

4.4

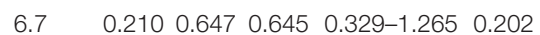

*Indicates that the patients' EGFR mutation status was assessable. NSCLC, non-small-cell lung cancer; KPS, Karnofsky performance status; AJCC, American Joint Committee on Cancer; EGFR, epidermal growth factor receptor; $L D H$, lactate dehydrogenase; ULN, upper limit of normal; $d N L R$, derived neutrophil to lymphocyte ratio; $\chi 2$, Chi-square statistic.

(19-21), respectively. Moreover, as second-line or later therapy, data from CheckMate 017 and CheckMate $057(5,6)$ also revealed that the PD-1/PD-L1 inhibitor alone had superior 
TABLE 5 | Incidence of adverse events (AEs).

\begin{tabular}{|c|c|c|c|}
\hline Treatment-related AEs, n (\%) & $\begin{array}{l}\text { Monotherapy } \\
\text { group }(n=26)\end{array}$ & $\begin{array}{l}\text { Combination } \\
\text { therapy group } \\
(n=58)\end{array}$ & $\boldsymbol{P}$ \\
\hline Any grade & $5(19.2 \%)$ & $11(18.9 \%)$ & 1.000 \\
\hline Fatigue & $0(0)$ & $1(1.7 \%)$ & \\
\hline Rash & $1(3.8 \%)$ & $1(1.7 \%)$ & \\
\hline Diarrhea & $1(3.8 \%)$ & $2(3.4 \%)$ & \\
\hline Decreased weight & $1(3.8 \%)$ & 0 & \\
\hline Decreased appetite & 0 & $1(1.7 \%)$ & \\
\hline Myocarditis & 0 & $4(6.8 \%)$ & \\
\hline Pneumonitis & $2(7.6 \%)$ & $4(6.8 \%)$ & \\
\hline Hypothyroidism & 0 & $1(1.7 \%)$ & \\
\hline Neutrophil count decreased & 0 & $3(5.1 \%)$ & \\
\hline Grade $\geq 3$ & $2(7.6 \%)$ & $6(10.2 \%)$ & 1.000 \\
\hline Neutrophil count decreased & 0 & $1(1.7 \%)$ & \\
\hline Pneumonitis & $1(3.8 \%)$ & $2(3.4 \%)$ & \\
\hline Myocarditis & 0 & $2(3.4 \%)$ & \\
\hline Diarrhea & 0 & $1(1.7 \%)$ & \\
\hline Decreased weight & $1(3.8 \%)$ & 0 & \\
\hline AEs leading to discontinuation & $1(3.8 \%)$ & $5(8.5 \%)$ & \\
\hline Pneumonitis & $1(3.8 \%)$ & $2(3.4)$ & \\
\hline Myocarditis & 0 & $2(3.4)$ & \\
\hline Diarrhea & 0 & $1(1.7 \%)$ & \\
\hline AEs leading to death & 0 & $1(1.7 \%)$ & \\
\hline Pneumonitis & 0 & $1(1.7 \%)$ & \\
\hline
\end{tabular}

$A E$, adverse event.

efficacy over docetaxel. However, whether chemotherapy plus PD-1/PD-L1 inhibitor as a second-line or later therapy is superior to $\mathrm{PD}-1 / \mathrm{PD}-\mathrm{L} 1$ inhibitor alone remained unclear. Our study revealed that addition of chemotherapy to PD-1 inhibitors did not improve OS or PFS compared with PD1/PD-L1 inhibitor alone for NSCLC patients as second-line and later therapy. PFS in the monotherapy group was longer than that in the PD-1 inhibitor plus chemotherapy group. Univariate and multivariate analyses suggested that male sex and $\mathrm{LDH} \leq \mathrm{ULN}$ were independent favorable factors for PFS. The incidence of grade 3-4 AEs in the combination therapy group was relatively higher than that in the monotherapy group, although there was no significant difference between the two groups. In the current study, one patient died of immune-related pneumonitis.

Notably, our study found that combination therapy group was superior to monotherapy group in the early stage of OS and PFS curves. In early stage of the survival curves of CheckMate 057, nivolumab was inferior to docetaxel (6). The analysis of early survival in CheckMate 057 indicated that death within the first three months of treatment was mainly due to disease progression (22). In the study of pretreated patients with NSCLC, the rates of hyperprogressive disease (HPD) of patients receiving PD-1/PD-L1 inhibitors and singleagent chemotherapy were 13.8 and $5.1 \%$, respectively (23). HPD mainly occurred within two months after receiving PD1/PD-L1 inhibitors and generally related to poor prognosis (23,
24). However, HPD in patients treated with immunotherapy plus chemotherapy was rarely reported. These differences are supported by our results. The combination therapy regimen may be able to reduce the risk of HPD related to PD-1/PDL1 inhibitors.

In our study, the PFS of the monotherapy group was longer than that of the combination therapy group, which was different from the result of a prior retrospective analysis that compared the efficacy of a PD-1 inhibitor plus chemotherapy and/or bevacizumab and PD-1 inhibitor alone for patients with advanced NSCLC in second-line or later therapy (25). The mPFS was reported at 7.5 and 3.3 months in the combination therapy and monotherapy groups, respectively $(P<0.001)$. These differences in results from our study might be related to the KPS score at the study initiation. Their rate of KPS $\geq 90$ in the combination therapy group was approximately double that in our combination therapy group ( 86.4 vs. $44.8 \%$ ). Although the KPS score in multivariate analysis had no impact on prognosis, advanced-stage patients undergoing second-line or later therapy often had lower KPS scores. The low KPS score indicates a poor tolerance to combination therapy. Moreover, we observed that patients in the monotherapy group had a longer PFS than those in the combination therapy group in the subgroup analysis of those receiving treatment beyond second-line therapy, while there was no significant difference between the two groups in the subgroup of second-line therapy. Although the subgroup analyses did not provide adequately powered evidence for efficacy comparison, they offer a direction for future studies to determine characteristics of those patients who may benefit from combination therapy.

The efficacy of PD-1/PD-L1 inhibitors in patients with tumors harboring EGFR mutations or ALK fusions is still conflicting. A small sample study suggested that uncommon EGFR mutations in tumors may be favorable prognostic factors for therapeutic effect of nivolumab in patients with NSCLC (26). However, in a retrospective analysis of 58 patients with NSCLC treated with $\mathrm{PD}-1 / \mathrm{PD}-\mathrm{L} 1$ inhibitors, the response rate was $3.6 \%$ in the EGFR mutation or ALK fusion subgroups and $23.3 \%$ in the wild-type subgroups (27). Currently, it is widely considered that PD-1/PD-L1 inhibitors have limited efficacy in patients with tumors harboring targetable mutations. A metaanalysis (28) including the CheckMate 057, KEYNOTE 010 and POPLAR trials indicated that PD-1/PD-L1 inhibitors alone did not show superior survival over docetaxel in patients with tumor EGFR mutations. Notably, in the IMMUNOTARGET study that evaluated the efficacy of PD-1/L1 inhibitors alone in patients with NSCLC with driver gene mutations, none of the twenty-three patients with ALK fusions had a disease response (29). Of the 84 patients involved in our study, 60 patients underwent somatic genetic testing. 9 of them had tumors which harbored EGFR mutations, while 2 had ALK fusions. Both patients with tumor EGFR mutations in the monotherapy group exhibited disease progression. In the combination therapy group, only one of the seven patients with tumor EGFR mutations had progressive disease, and the other six patients had stable disease. In the population 
with tumor ALK fusion, two patients received combination therapy, and both had progressive disease after two cycles. It is possible that patients with tumor EGFR mutations who exhibit disease progression after first-line therapy may benefit from PD-1 inhibitors plus chemotherapy. However, in the ALK fusion population, this survival benefit from PD-1/L1 inhibitors may be limited.

Lactate dehydrogenase, as a systemic inflammation indicator, has attracted attention. Multiple previous studies in various cancer types indicated that high LDH level is a poor prognostic factor for PFS or OS (30-33). A meta-analysis of 1136 patients with NSCLC treated with immune checkpoint inhibitors suggested that high LDH levels were related to shorter PFS and OS (30). Moreover, combination of dNLR and LDH levels as lung immune prognostic index (LIPI) is better at predicting these effects of PD-1/PD-L1 inhibitors $(17,18)$. The present study also confirmed that $\mathrm{LDH} \leq \mathrm{ULN}$ was a favorable prognostic factor for PFS in patients with pre-treated NSCLC, which was consistent with the findings of a previous study (30). Our study also suggested that male sex was a favorable prognostic factor for PFS. A meta-analysis including 20 randomized trials suggested that males benefit more than females, although the OS for both sexes can be improved by PD-1 inhibitors (34). These differences may be related to poor immunity $(35,36)$, high tumor mutation burden (TMB) (37) and the influence of smoking behavior on TMB in males (38). The present study's real-world results were similar to those of previous reports and support further research in related directions, which favor selecting a population who would benefit from PD-1/PDL1 inhibitors.

Although there was no significant difference between the two groups, the incidence of grade 3-4 AEs was relatively higher in patients receiving combination therapy. Myocarditis and pneumonitis, as the primary reasons leading to treatment interruption in the combination therapy group, are noteworthy. In our study, the incidence of grade 3-4 AEs was 7.6\% in the monotherapy group and $10.2 \%$ in the combination therapy group. The incidence of any grade AEs in our study was underestimated, perhaps because low-grade AEs were not obvious for patients to actively report. A meta-analysis reported by Nishino et al. showed that the incidence of pneumonitis in NSCLC was $4.1 \%$ (39). The most common antiPD-1/PD-L1-related fatalities were from pneumonitis in a metaanalysis predominantly consisting of NSCLC and melanoma (40). The incidence of myocarditis of any grade was only $0.06 \%$, but the mortality of high-grade myocarditis can reach $36 \%$ in patients who received PD-1/PD-L1 inhibitors alone $(41,42)$. In patients receiving a combination of anti-PD1/PD-L1 with anti-CTLA-4 inhibitors, the fatality rate can even reach $67 \%$ (42). In our study, the patient who died of pneumonitis in the combination therapy group had a history of chronic obstructive pulmonary disease, which might be a risk factor for pneumonitis. These two specific AEs should be noted in patients treated with PD-1/PD-L1 inhibitors plus chemotherapy.

In our study, patients who previously received immunotherapy were excluded. This restriction was mainly due to two reasons. First, whether it is beneficial to continue to use PD-1/PD-L1 inhibitors beyond immunotherapy progression is still unknown. Although previous studies have reported that patients with immunotherapy progression may still receive benefit from immunotherapy $(43,44)$, no relevant study has compared the efficacy between continuing PD-1/PD-L1 inhibitor and switching to another therapy later. The latest version of the NCCN guidelines recommend chemotherapy alone or ramucirumab plus docetaxel rather than nivolumab, pembrolizumab and atezolizumab as subsequent therapy if the disease has progressed on PD-1/PD-L1 inhibitor therapy (45). Second, previous immunotherapy potentially influences the decision to use immunotherapy combined with chemotherapy later. If disease progressed on immunotherapy, patients may prefer to receive combination therapy subsequently to allow a higher possibility of disease response. Therefore, previously treated patients would be expected to be present in the combination therapy group. To avoid this potential bias, we did not include patients who had previously received immunotherapy in this study.

As a retrospective analysis, our study has some limitations. First, the small sample size influenced the statistical power and may lead to selection bias and measurement bias. Because of the small sample size, the conclusion is not representative of the whole population. Despite adjustment by the Cox regression model, confounding factors may still exist. Further analysis with a larger sample size is necessary in the future. Moreover, a previous study has reported the rate of EGFR mutations in non-small cell lung cancer in never-smoking Asian patients to be $60.7 \%$, while the rate of EGFR mutations in our study was only $20 \%$ (46). Selection bias may be a major reason for this difference. In the EGFRmutated NSCLC, targeted therapy is the preferred therapeutic regimen, and immunotherapy is rarely used because of reported lower rate of benefit. Thus, patients whose tumors do not harbor EGFR mutations may preferentially be treated with immunotherapy, which may explain the lower rates of somatic EGFR mutations in our study. Second, the available baseline features in our retrospective study were limited. Some important clinical information, which may affect the survival time and treatment response, such as PD-L1 tumor proportion score or TMB level and baseline lung function, was not available for all patients.

\section{CONCLUSION}

In conclusion, our study suggested that the addition of chemotherapy to PD-1 inhibitors did not improve clinical outcomes compared to monotherapy with PD-1/PD-L1 inhibitors in second-line or later treatment settings for patients with advanced NSCLC. Our observations provide directions for future treatment studies and valuable clues about prognostic factors and adverse events with use of PD-1/PD-L1 inhibitors with or without chemotherapy in this patient population. 


\section{DATA AVAILABILITY STATEMENT}

The datasets generated for this study are available on request to the corresponding author.

\section{ETHICS STATEMENT}

The studies involving human participants were reviewed and approved by the Ethics Committee of Shandong Cancer Hospital Affiliated to Shandong First Medical University. The patients/participants provided their written informed consent to participate in this study.

\section{REFERENCES}

1. Bray F, Ferlay J, Soerjomataram I, Siegel RL, Torre LA, Jemal A. Global cancer statistics 2018: GLOBOCAN estimates of incidence and mortality worldwide for 36 cancers in 185 countries. CA Cancer J Clin. (2018) 68:394-424. doi: 10.3322/caac. 21492

2. American Cancer Society. Types of lung cancer. (2020). Available online at: https://www.cancer.org/cancer/lungcancer/about/what-is.html (accessed February 10, 2020).

3. Fossella FV, DeVore R, Kerr RN, Crawford J, Natale RR, Dunphy F, et al. Randomized phase III trial of docetaxel versus vinorelbine or ifosfamide in patients with advanced non-small-cell lung cancer previously treated with platinum-containing chemotherapy regimens. The TAX 320 non-small cell lung cancer study group. J Clin Oncol. (2000) 18:2354-62. doi: 10.1200/JCO. 2000.18.12.2354

4. Shepherd FA, Dancey J, Ramlau R, Mattson K, Gralla R, O’Rourke M, et al. Prospective randomized trial of docetaxel versus best supportive care in patients with non-small-cell lung cancer previously treated with platinumbased chemotherapy. J Clin Oncol. (2000) 18:2095-103. doi: 10.1200/JCO. 2000.18.10.2095

5. Brahmer J, Reckamp KL, Baas P, Crino L, Eberhardt WE, Poddubskaya E, et al. Nivolumab versus docetaxel in advanced squamous-cell non-small-cell lung cancer. N Engl J Med. (2015) 373:123-35. doi: 10.1056/NEJMoa1504627

6. Borghaei H, Paz-Ares L, Horn L, Spigel DR, Steins M, Ready NE, et al. Nivolumab versus docetaxel in advanced nonsquamous non-small-cell lung cancer. N Engl J Med. (2015) 373:1627-39. doi: 10.1056/NEJMoa1507643

7. Herbst RS, Baas P, Kim DW, Felip E, Perez-Gracia JL, Han JY, et al. Pembrolizumab versus docetaxel for previously treated, PD-L1-positive, advanced non-small-cell lung cancer (KEYNOTE-010): a randomised controlled trial. Lancet. (2016) 387:1540-50. doi: 10.1016/S0140-6736(15) 01281-7

8. Rittmeyer A, Barlesi F, Waterkamp D, Park K, Ciardiello F, von Pawel J, et al. Atezolizumab versus docetaxel in patients with previously treated nonsmall-cell lung cancer (OAK): a phase 3, open-label, multicentre randomised controlled trial. Lancet. (2017) 389:255-65. doi: 10.1016/S0140-6736(16) 32517-X

9. Gettinger S, Borghaei H, Brahmer J, Chow L, Burgio M, De Castro Carpeno $\mathrm{J}$, et al. OA14.04 five-year outcomes from the randomized, phase 3 trials checkmate 017/057: Nivolumab vs Docetaxel in previously treated NSCLC. J Thorac Oncol. (2019) 14:S244-5. doi: 10.1016/j.jtho.2019.08.486

10. Ramakrishnan R, Huang C, Cho HI, Lloyd M, Johnson J, Ren X, et al. Autophagy induced by conventional chemotherapy mediates tumor cell sensitivity to immunotherapy. Cancer Res. (2012) 72:5483-93. doi: 10.1158/ 0008-5472.CAN-12-2236

11. Casares N, Pequignot MO, Tesniere A, Ghiringhelli F, Roux S, Chaput N, et al. Caspase-dependent immunogenicity of doxorubicin-induced tumor cell death. J Exp Med. (2005) 202:1691-701. doi: 10.1084/jem.20050915

12. Zhang L, Dermawan K, Jin M, Liu R, Zheng H, Xu L, et al. Differential impairment of regulatory $\mathrm{T}$ cells rather than effector $\mathrm{T}$ cells by paclitaxel-based

\section{AUTHOR CONTRIBUTIONS}

HZ designed the study. XZ collected the data of clinical trials together with SX and MW and drafted the manuscript. XJ, JL, and YT coordinated. All authors read and approved the final manuscript.

\section{FUNDING}

This work was supported by the National Natural Science Foundation of China (Grant No. 81972862 to HZ).

chemotherapy. Clin Immunol. (2008) 129:219-29. doi: 10.1016/j.clim.2008.07. 013

13. Le HK, Graham L, Cha E, Morales JK, Manjili MH, Bear HD. Gemcitabine directly inhibits myeloid derived suppressor cells in BALB/c mice bearing $4 \mathrm{~T} 1$ mammary carcinoma and augments expansion of T cells from tumor-bearing mice. Int Immunopharmacol. (2009) 9:900-9. doi: 10.1016/j.intimp.2009.03. 015

14. Apetoh L, Ghiringhelli F, Tesniere A, Obeid M, Ortiz C, Criollo A, et al. Tolllike receptor 4-dependent contribution of the immune system to anticancer chemotherapy and radiotherapy. Nat Med. (2007) 13:1050-9. doi: 10.1038/ nm1622

15. Gandhi L, Rodriguez-Abreu D, Gadgeel S, Esteban E, Felip E, De Angelis F, et al. Pembrolizumab plus chemotherapy in metastatic non-small-cell lung cancer. N Engl J Med. (2018) 378:2078-92. doi: 10.1056/NEJMoa1801005

16. Reck M, Rodriguez-Abreu D, Robinson AG, Hui R, Csoszi T, Fulop A, et al. Pembrolizumab versus chemotherapy for PD-L1-positive non-small-cell lung cancer. N Engl J Med. (2016) 375:1823-33. doi: 10.1056/NEJMoa1606774

17. Kazandjian D, Gong Y, Keegan P, Pazdur R, Blumenthal GM. Prognostic value of the lung immune prognostic index for patients treated for metastatic nonsmall cell lung cancer. JAMA Oncol. (2019) 5:1481-5. doi: 10.1001/jamaoncol. 2019.1747

18. Mezquita L, Auclin E, Ferrara R, Charrier M, Remon J, Planchard D, et al. Association of the lung immune prognostic index with immune checkpoint inhibitor outcomes in patients with advanced non-small cell lung cancer. JAMA Oncol. (2018) 4:351-7. doi: 10.1001/jamaoncol.2017.4771

19. Paz-Ares L, Luft A, Vicente D, Tafreshi A, Gumus M, Mazieres J, et al. Pembrolizumab plus chemotherapy for squamous non-small-cell lung cancer. N Engl J Med. (2018) 379:2040-51. doi: 10.1056/NEJMoa1810865

20. Socinski MA, Jotte RM, Cappuzzo F, Orlandi F, Stroyakovskiy D, Nogami $\mathrm{N}$, et al. Atezolizumab for first-line treatment of metastatic nonsquamous NSCLC. N Engl J Med. (2018) 378:2288-301. doi: 10.1056/NEJMoa1716948

21. Gadgeel S, Rodriguez-Abreu D, Speranza G, Esteban E, Felip E, Domine M, et al. Updated analysis from KEYNOTE-189: pembrolizumab or placebo plus pemetrexed and platinum for previously untreated metastatic nonsquamous non-small-cell lung cancer. J Clin Oncol. (2020) 38:1505-17. doi: 10.1200/JCO. 19.03136

22. Peters S, Cappuzzo F, Horn L, Paz-Ares L, Borghaei H, Barlesi F, et al. OA03.05 analysis of early survival in patients with advanced non-squamous nsclc treated with nivolumab vs docetaxel in checkmate 057. J Thorac Oncol. (2017) 12:S253.

23. Ferrara R, Mezquita L, Texier M, Lahmar J, Audigier-Valette C, Tessonnier $\mathrm{L}$, et al. Hyperprogressive disease in patients with advanced non-small cell lung cancer treated with PD-1/PD-L1 inhibitors or with single-agent chemotherapy. JAMA Oncol. (2018) 4:1543-52. doi: 10.1001/jamaoncol.2018. 3676

24. Champiat S, Dercle L, Ammari S, Massard C, Hollebecque A, Postel-Vinay $\mathrm{S}$, et al. Hyperprogressive disease is a new pattern of progression in cancer patients treated by anti-PD-1/PD-L1. Clin Cancer Res. (2017) 23:1920-8. doi: 10.1158/1078-0432.CCR-16-1741 
25. Zhang F, Huang D, Li T, Zhang S, Wang J, Zhang Y, et al. Anti-PD-1 therapy plus chemotherapy and/or bevacizumab as second line or later treatment for patients with advanced non-small cell lung cancer. J Cancer. (2020) 11:741-9. doi: 10.7150/jca.37966

26. Yoshida H, Kim YH, Ozasa H, Nagai H, Sakamori Y, Tsuji T, et al. Nivolumab in non-small-cell lung cancer with EGFR mutation. Ann Oncol. (2018) 29:7778. doi: 10.1093/annonc/mdx745

27. Gainor JF, Shaw AT, Sequist LV, Fu X, Azzoli CG, Piotrowska Z, et al. EGFR mutations and ALK rearrangements are associated with low response rates to PD-1 pathway blockade in non-small cell lung cancer: a retrospective analysis. Clin Cancer Res. (2016) 22:4585-93. doi: 10.1158/1078-0432.CCR-15-3101

28. Lee CK, Man J, Lord S, Links M, Gebski V, Mok T, et al. Checkpoint inhibitors in metastatic EGFR-mutated non-small cell lung cancer-A meta-analysis. $J$ Thorac Oncol. (2017) 12:403-7. doi: 10.1016/j.jtho.2016.10.007

29. Mazieres J, Drilon A, Lusque A, Mhanna L, Cortot AB, Mezquita L, et al. Immune checkpoint inhibitors for patients with advanced lung cancer and oncogenic driver alterations: results from the IMMUNOTARGET registry. Ann Oncol. (2019) 30:1321-8. doi: 10.1093/annonc/mdz167

30. Zhang Z, Li Y, Yan X, Song Q, Wang G, Hu Y, et al. Pretreatment lactate dehydrogenase may predict outcome of advanced non small-cell lung cancer patients treated with immune checkpoint inhibitors: a meta-analysis. Cancer Med. (2019) 8:1467-73. doi: 10.1002/cam4.2024

31. Diem S, Kasenda B, Spain L, Martin-Liberal J, Marconcini R, Gore M, et al. Serum lactate dehydrogenase as an early marker for outcome in patients treated with anti-PD-1 therapy in metastatic melanoma. Br J Cancer. (2016) 114:256-61. doi: 10.1038/bjc.2015.467

32. Mori K, Kimura S, Parizi MK, Enikeev DV, Glybochko PV, Seebacher V, et al. Prognostic value of lactate dehydrogenase in metastatic prostate cancer: a systematic review and meta-analysis. Clin Genitourin Cancer. (2019) 17:40918. doi: 10.1016/j.clgc.2019.07.009

33. Shen J, Chen Z, Zhuang Q, Fan M, Ding T, Lu H, et al. Prognostic value of serum lactate dehydrogenase in renal cell carcinoma: a systematic review and meta-analysis. PLoS One. (2016) 11:e0166482. doi: 10.1371/journal.pone. 0166482

34. Conforti F, Pala L, Bagnardi V, De Pas T, Martinetti M, Viale G, et al. Cancer immunotherapy efficacy and patients' sex: a systematic review and metaanalysis. Lancet Oncol. (2018) 19:737-46. doi: 10.1016/S1470-2045(18)302614

35. Klein SL, Flanagan KL. Sex differences in immune responses. Nat Rev Immunol. (2016) 16:626-38. doi: 10.1038/nri.2016.90

36. Pennell LM, Galligan CL, Fish EN. Sex affects immunity. J Autoimmun. (2012) 38:J282-91. doi: 10.1016/j.jaut.2011.11.013

37. Xiao D, Pan H, Li F, Wu K, Zhang X, He J. Analysis of ultra-deep targeted sequencing reveals mutation burden is associated with gender and clinical outcome in lung adenocarcinoma. Oncotarget. (2016) 7:22857-64. doi: 10. 18632/oncotarget.8213
38. Rizvi NA, Hellmann MD, Snyder A, Kvistborg P, Makarov V, Havel JJ, et al. Cancer immunology. Mutational landscape determines sensitivity to PD-1 blockade in non-small cell lung cancer. Science. (2015) 348:124-8. doi: 10. 1126/science.aaa1348

39. Nishino M, Giobbie-Hurder A, Hatabu H, Ramaiya NH, Hodi FS. Incidence of programmed cell death 1 inhibitor-related pneumonitis in patients with advanced cancer: a systematic review and meta-analysis. JAMA Oncol. (2016) 2:1607-16. doi: 10.1001/jamaoncol.2016.2453

40. Wang DY, Salem JE, Cohen JV, Chandra S, Menzer C, Ye F, et al. Fatal toxic effects associated with immune checkpoint inhibitors: a systematic review and meta-analysis. JAMA Oncol. (2018) 4:1721-8. doi: 10.1001/jamaoncol.2018. 3923

41. Johnson DB, Balko JM, Compton ML, Chalkias S, Gorham J, Xu Y, et al. Fulminant myocarditis with combination immune checkpoint blockade. $N$ Engl J Med. (2016) 375:1749-55. doi: 10.1056/NEJMoa1609214

42. Moslehi JJ, Salem JE, Sosman JA, Lebrun-Vignes B, Johnson DB. Increased reporting of fatal immune checkpoint inhibitor-associated myocarditis. Lancet. (2018) 391:933. doi: 10.1016/S0140-6736(18) 30533-6

43. Stinchcombe TE, Miksad RA, Gossai A, Griffith SD, Torres AZ. Real-world outcomes for advanced non-small cell lung cancer patients treated with a PD-L1 inhibitor beyond progression. Clin Lung Cancer. (2020) 21:389-94.e3. doi: 10.1016/j.cllc.2020.04.008

44. Gandara DR, von Pawel J, Mazieres J, Sullivan R, Helland A, Han JY, et al. Atezolizumab treatment beyond progression in advanced NSCLC: results from the randomized, phase III OAK study. J Thorac Oncol. (2018) 13:190618. doi: 10.1016/j.jtho.2018.08.2027

45. National Comprehensive Cancer Network. Non-Small Cell Lung Cancer Clinical Practice Guidelines. (2020). Available online at: https://www.nccn.org/ professionals/physician_gls/pdf/nscl.pdf (accessed June 30, 2020).

46. Shi Y, Au JS, Thongprasert S, Srinivasan S, Tsai CM, Khoa MT, et al. A prospective, molecular epidemiology study of EGFR mutations in Asian patients with advanced non-small-cell lung cancer of adenocarcinoma histology (PIONEER). J Thorac Oncol. (2014) 9:154-62. doi: 10.1097/JTO. 0000000000000033

Conflict of Interest: The authors declare that the research was conducted in the absence of any commercial or financial relationships that could be construed as a potential conflict of interest.

Copyright (C) 2020 Zhai, Jing, Li, Tian, Xu, Wang and Zhu. This is an open-access article distributed under the terms of the Creative Commons Attribution License (CC BY). The use, distribution or reproduction in other forums is permitted, provided the original author(s) and the copyright owner(s) are credited and that the original publication in this journal is cited, in accordance with accepted academic practice. No use, distribution or reproduction is permitted which does not comply with these terms. 